\title{
Use of CA15-3 for screening breast cancer: An antibody-lectin sandwich assay for detecting glycosylation of CA15-3 in sera
}

\author{
JAE WOONG CHOI ${ }^{1 *}$, BYUNG-IN MOON $^{2 *}$, JUN WOO LEE $^{2}$, HYOUNG JIN KIM $^{1}$, \\ YINGJI JIN $^{1}$ and HONG-JIN KIM ${ }^{1}$ \\ ${ }^{1}$ Laboratory of Virology, College of Pharmacy, Chung-Ang University, Seoul 06974; \\ ${ }^{2}$ Breast and Thyroid Cancer Center, Ewha Womans University Cancer Center for Women, \\ Seoul 07985, Republic of Korea
}

Received October 6, 2017; Accepted May 3, 2018

DOI: $10.3892 /$ or.2018.6433

\begin{abstract}
Elevated serum CA15-3 assessed by enzyme-linked immunosorbent assay (ELISA) has been considered a diagnostic marker of breast cancer. However, accumulating data indicate that the current ELISA system for detecting CA15-3, which targets the peptide backbone of CA15-3, is not sufficiently sensitive to detect early or localized breast cancer. In the present study, we designed an antibody-lectin sandwich assay detecting glycosylation of CA15-3 in patients with breast cancer. Immobilized anti-CA15-3 monoclonal antibody captures CA15-3 in serum, and glycosylation of the CA15-3 is detected with Concanavalin A (ConA) lectin, which preferentially bind high-mannose $\mathrm{N}$-glycans. ConA provided the best signal for detecting serum CA15-3 among 9 types of lectin, Since CA15-3 is a heavily glycosylated protein, detecting the glycosylation of CA15-3 should be a much more sensitive way to assess CA15-3 than the current ELISA method. Linear responses were obtained in the anti-CA15-3 antibody-ConA sandwich assay when sera were diluted up to 2000-fold. This dilution factor is comparable with that of the current ELISA system which allows 50- to 100-fold serum dilutions. The glycosylation level of CA15-3 was found to increase with increasing breast cancer stage in the sandwich assay. The assay system appeared to efficiently discriminate breast cancer stage I (sensitivity: 63\%, specificity: 69\%), IIA (sensitivity: 77\%, specificity: 75\%), IIB (sensitivity: 69\%, specificity: $86 \%$ ) and III (sensitivity: $80 \%$, specificity: $65 \%$ ) from benign breast disease. The antibody-lectin sandwich
\end{abstract}

Correspondence to: Professor Hong-Jin Kim, Laboratory of Virology, College of Pharmacy, Chung-Ang University, 84 Heukseok-Ro, Dongjak-Gu, Seoul 06974, Republic of Korea

E-mail: hongjink@cau.ac.kr

${ }^{*}$ Contributed equally

Key words: breast cancer, biomarker, CA15-3, lectin, MUC1, glycosylation assay shows promise as a new prospect for the early detection of breast cancer.

\section{Introduction}

Breast cancer is the most common cancer among women and the second most common cancer worldwide (1). It has been estimated that there were 1.7 million new cases and 521,900 deaths due to the disease in 2012; this corresponds to $25 \%$ of all new cancer cases and $15 \%$ of cancer deaths among women, respectively (2). Family history, environment and stress are all somehow implicated in the development of breast cancer (3). The 5-year survival of women with breast cancer is highly dependent on tumor stage: that of women with stage 0 or I is $98 \%$ and those of stages II and III are 85 and $60 \%$, respectively (4). However, the 5-year survival of stage IV is only $20 \%$ (4). Early detection of breast cancer can provide increased treatment options as well as improved conditions for treatment or surgery.

Diagnostic techniques including mammography, magnetic resonance imaging, ultrasound, computerized tomography, positron emission tomography and biopsy have been used for detecting breast cancer (5). These strategies are expensive, time-consuming and cannot cope with large numbers of patients at the same time. Cancer screening using serum biomarkers would be an ideal diagnostic technique since cancer could be detected by routine health examination. Moreover, such screening could increase the rate of detection of early stage cancer and eventually lead to increased survival.

CA15-3 is a soluble form of MUC1, a transmembrane protein that possesses variable numbers of tandem repeats of peptides modified by glycosylation, and the most extensively studied serum biomarker for breast cancer (6). The current system for detecting serum CA15-3 is a sandwich enzyme-linked immunosorbent assay (ELISA) using two types of monoclonal anti-CA15-3 antibodies (115D8: capture antibody; DF3: detection antibody) (7,8). Attempts to use ELISA systems in the screening of breast cancer over the past three decades have indicated that the serum CA15-3 level is not suitable for early detection of breast cancer since its level rarely increases in patients with early or localized breast cancer (7). 
CA15-3 remains useful for monitoring the effects of treatment in patients with metastatic breast cancer (9). Currently, the American Society of Clinical Oncology does not recommend use of CA15-3 for screening, diagnosis, staging, or routine surveillance of breast cancer (10).

Changes in glycosylation are a hallmark of cancer progression. Numerous studies suggest that aberrant glycosylation is a sensitive indicator of carcinogenesis (11). Cancer-related changes in glycosylation are thought to involve altered expression of glycosyltransferase and chaperone genes, and mislocalization of glycosyltransferases (11). Most analyses of glycosylation have involved highly purified glycoproteins and expensive procedures such as mass spectrometry, capillary electrophoresis and high-performance liquid chromatography. These are not suitable for routine examination although they provide detailed glycosylation profiles.

Since CA15-3 is a heavily glycosylated protein (12), glycosylation changes have great potential for reflecting carcinogenesis, and previous studies have indeed pointed to glycosylation changes of CA15-3 in breast cancer (13). Antibody-lectin sandwich assays have been suggested as platforms for examining glycosylation in non-purified serum samples (14). In such assays, the antibody against the target glycoprotein immobilized on 96-well plates captures the serum glycoprotein, and glycosylation of the glycoprotein is detected with a lectin (15). There is some evidence that antibody-lectin sandwich assays can be used for cancer screening $(16,17)$. However, the capture antibody is also glycosylated and this can interfere with detection of the target glycosylation signal. Also, glycoproteins contained in blocking agents can inhibit the target signal.

In the present study, we developed an antibody-lectin sandwich assay for detecting glycosylation of CA15-3 in non-purified serum samples that was designed to overcome these technical issues. Our results indicated that this strategy is useful for screening early or localized breast cancer.

\section{Materials and methods}

Ethical approval. This study was carried out with the approval of the Ewha Womans University Mokdong Hospital Institutional Review Board (Seoul, Korea) and was conducted in accordance with the Declaration of Helsinki. Serum samples of patients were collected after obtaining written informed consents.

Specimens. Samples were collected in a prospective and consecutive manner. Sera from women diagnosed with benign breast disease $(n=47)$ and breast cancer stages $0(n=6)$, I $(n=48)$, IIA $(n=36)$, IIB $(n=12)$ and III $(n=26)$ were collected from November 2015 to January 2017 at the Breast and Thyroid Cancer Center of Ewha Womans University Cancer Center for Women. The mean age of the benign group was 40, and those of breast cancer stage 0, I, IIA, IIB and III were 49, $51,50,55$ and 54, respectively. The age range of the benign group was 19-74, and those of breast cancer stage 0, I, IIA, IIB and III were 25-76, 29-78, 29-75, 33-83 and 36-78, respectively. The breast cancer stage was determined according to the tumor-node-metastasis (TNM) staging system. Serum samples were collected before surgery or treatment. Each serum sample was centrifuged at $12,000 \mathrm{x} \mathrm{g}$ for $10 \mathrm{~min}$, aliquoted and stored at $-80^{\circ} \mathrm{C}$ until use, and a vial set was used within a few days after thawing.

Investigation of the effects of oxidation of the coating antibody and blocking agent. Immunoplates (96-wells) (Greiner Bio-One, Kremsmünster, Austria) were coated with mouse anti-CA15-3 monoclonal antibody (100 ng/well; vol. $100 \mu \mathrm{l}$; cat. no. 10-C03E; Fitzgerald Industries International, Acton, MA, USA) or regular fetal bovine serum (FBS; vol. $100 \mu \mathrm{l}$; GenDEPOT, Barker, TX, USA) at $4^{\circ} \mathrm{C}$ for $16 \mathrm{~h}$. The anti-CA15-3 monoclonal antibody and FBS were prepared in phosphate-buffered saline (PBS) at $1 \mu \mathrm{g} / \mathrm{ml}$ and $10 \%(\mathrm{v} / \mathrm{v})$, respectively. The coated wells were reacted with $320 \mu \mathrm{l}$ oxidation buffer [50 mM sodium acetate (Duksan Pure Chemicals, Co., Ltd., Ansan, Korea), $50 \mathrm{mM}$ sodium metaperiodate (Sigma-Aldrich; Merck, St. Louis, MO, USA) pH 4.0] for $1 \mathrm{~h}$ at room temperature. Control wells were reacted with Tris-buffered saline (TBS) containing 1\% Tween-20 (TBS-T) instead of oxidation buffer. The plates were blocked with TBS-T for $3 \mathrm{~h}$ at room temperature (reaction vol. $320 \mu \mathrm{l}$ ). Twelve types of biotinylated lectin were prepared in TBS-T at final concentrations of $1 \mu \mathrm{g} / \mathrm{ml}$ and incubated on the plates for $1 \mathrm{~h}$ at room temperature (reaction vol. $100 \mu \mathrm{l}$ ). All the lectins were purchased from Vector Laboratories, Inc. (Burlingame, CA, USA). Lectins bound to the coated antibody or FBS were detected with poly-horseradish peroxidase (HRP)-conjugated streptavidin (Pierce; Thermo Fisher Scientific, Inc., Waltham, MA, USA; 1/10,000 dilution; reaction vol. $100 \mu \mathrm{l}$ ). The plates were washed three to five times using TBS-T between reactions. Color reactions were developed with $o$-phenylenediamine (Sigma-Aldrich; Merck) and measured at $492 \mathrm{~nm}$.

Antibody-lectin sandwich assay for the detection of the glycosylation of CA15-3. Immunoplates (96 wells) were coated with mouse anti-CA15-3 monoclonal antibody (100 ng/well, vol. $100 \mu \mathrm{l}$ ) in PBS for $16 \mathrm{~h}$ at $4^{\circ} \mathrm{C}$, then blocked with $10 \% \mathrm{FBS}$ in TBS-T $(320 \mu \mathrm{l})$ for $3 \mathrm{~h}$ at room temperature. The coating antibody and FBS were oxidized using the oxidation buffer (320 $\mu \mathrm{l}$ ) aforementioned to block lectin binding. The wells were further blocked with $1 \%$ oxidized bovine serum albumin (oBSA) in TBS-T for $2 \mathrm{~h}$ at room temperature (reaction vol. $320 \mu \mathrm{l}$ ). oBSA was prepared as previously described (18). BSA was dissolved at a final concentration of $5 \%$ in the oxidation buffer, maintained at $4^{\circ} \mathrm{C}$ for $2 \mathrm{~h}$ and dialyzed against TBS-T for 2 days to remove the sodium metaperiodate. Sera were diluted $1 / 3,000$ or $1 / 4,000$ using $1 \%$ oBSA in TBS-T and reacted in the wells for $2 \mathrm{~h}$ at $37^{\circ} \mathrm{C}$ (reaction vol. $100 \mu \mathrm{l}$ ). Biotinylated lectins were prepared at a concentration of $1 \mu \mathrm{g} / \mathrm{ml}$ in TBS-T and reacted in the wells for $1 \mathrm{~h}$ at room temperature (reaction vol. $100 \mu \mathrm{l}$ ). Thereafter lectin bound to the CA15-3 glycan was detected with poly-HRP-conjugated streptavidin (reaction vol. $100 \mu \mathrm{l}$ ). Color was developed as aforementioned. The plates were washed three to five times using TBS-T between reactions, and seven washes were carried out prior to the color reaction.

A mirror plate, the coating antibody reaction which was omitted, was prepared, to check the non-specific reaction of the serum. The reaction of the mirror plate included blocking, oxidation, serum reaction, lectin reaction and streptavidin 


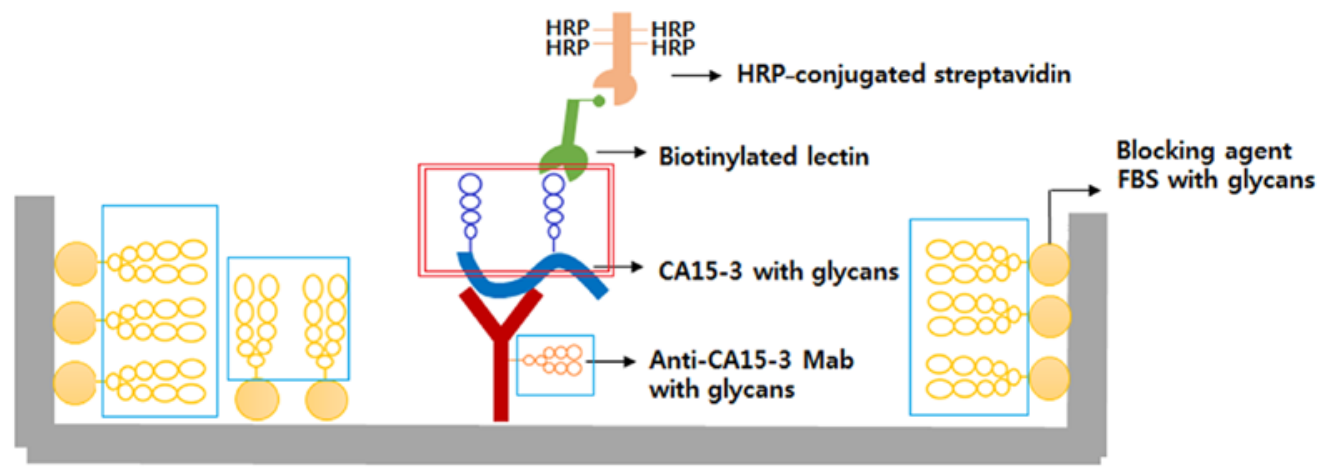

Well

Figure 1. Schematic diagram of the antibody-lectin sandwich assay. Immobilized anti-CA15-3 antibody captures CA15-3 in serum, and glycosylation of CA15-3 is detected with a biotinylated lectin followed by HRP-conjugated streptavidin. Blues boxes represent glycosyls of the coated anti-CA15-3 monoclonal antibody, and of FBS used as a blocking agent. The red boxes represent glycans of CA15-3.

reaction steps. The values of the anti-CA15-3-ConA sandwich assays were calculated as follows: optical density of the full series of reactions-optical density of the mirror plate reaction.

Sandwich ELISA for the detection of CA15-3. Immunoplates (96 wells) were coated with mouse anti-CA15-3 monoclonal antibody (50 ng/well) and blocked with 5\% skim milk at room temperate for $3 \mathrm{~h}$. Skim milk was prepared in phosphate-buffered saline (PBS) containing $0.05 \%$ Tween-20 (PBS-T). Then the plates were reacted with serum samples (1:100 dilution ratio) prepared in $0.5 \%$ skim milk in PBS-T at $37^{\circ} \mathrm{C}$ for $2 \mathrm{~h}$. The captured CA15-3 was detected using rabbit anti-MUC1 polyclonal antibody (1:3,000 dilution ratio; reaction: $37^{\circ} \mathrm{C}$ for $1 \mathrm{~h}$; Sino Biological, Inc., Beijing, China; cat. no. 12123-T24), together with HRP-conjugated anti-rabbit IgG polyclonal antibody (1:5,000 dilution ratio; reaction: $37^{\circ} \mathrm{C}$ for 40 min, Bethyl Laboratories, Inc., Montgomery, TX, USA; cat. no. A120-100P). The antibodies used to detect CA15-3 were prepared in $0.5 \%$ skim milk in PBST-T. The plates were washed three to five times using PBS-T between reactions. Color reactions were developed with $o$-phenylenediamine and measured at $492 \mathrm{~nm}$.

Immunoprecipitation of serum CA15-3. The serum sample was diluted 1:10 with PBS and reacted with rabbit anti-MUC1 polyclonal antibody (Sino Biological, Inc.; cat. no. 12123-T24) coupled with protein A agarose beads (Sigma-Aldrich; Merck) for $16 \mathrm{~h}$ at $4^{\circ} \mathrm{C}$. The protein A agarose beads were mixed with 5\% BSA in PBS to block non-specific reactions. The protein A agarose beads were then washed three times with PBS-T by centrifugation at $12,000 \mathrm{xg}$ for $10 \mathrm{~min}$, and the immunoprecipitates were collected by centrifugation as aforementioned and analyzed by western or lectin blotting.

Western blotting for CA15-3. Immunoprecipitates were diluted 1:10, fractionated on $12.5 \%$ polyacrylamide gels, and transferred onto polyvinylidene difluoride membranes (EMD Millipore, Burlington, MA, USA), and the membranes were blocked with 5\% skim milk (Bioworld Technology, Inc., St. Louis Park, MN, USA) in TBS-T. CA15-3 on the polyvinylidene difluoride membranes was detected using rabbit anti-MUC1 polyclonal antibody (1:1,000 dilution ratio), together with HRP-conjugated anti-rabbit $\operatorname{IgG}(1: 5,000)$. CA15-3 and anti-CA15-3 antibody bands were visualized on X-ray film (Kodak, USA) using an enhanced chemiluminescence kit (AbClon, Seoul, Korea).

Lectin blotting for detecting CA15-3 glycosylation. Immunoprecipitates were diluted 1:100, fractionated and transferred onto polyvinylidene difluoride membranes as aforementioned. The membranes were blocked with $5 \%$ oBSA in TBS-T for $4 \mathrm{~h}$ at room temperature with shaking overnight. The mannosylated $N$-glycosylation of CA15-3 was detected with biotinylated ConA $(1 \mu \mathrm{g} / \mathrm{ml})$, followed by poly-HRP-conjugated streptavidin $(1: 10,000)$. The bands were visualized as previously described. ConA and streptavidin were prepared in $0.5 \%$ oBSA in TBS-T.

Statistical analysis. Kruskal-Wallis or Mann-Whitney U test was used to evaluate differences in the glycosylation level of CA15-3 between groups, and $\mathrm{P}<0.05$ was considered significant. P-values, receiver operating characteristic (ROC) curves and area under the curve (AUC) values were obtained using GraphPad prism version 5.01 (GraphPad Software, Inc., La Jolla, CA, USA), and sensitivity and specificity were determined from the ROC curves: cut-offs were selected to maximize the sum of the sensitivity and specificity. Specificity $=$ number of true negatives $x$ 100/number of true negatives + number of false positives. Sensitivity $=$ number of true positives x 100/number of true positives + number of false negatives. Correlation between the mannosylated $N$-glycosylation level of CA15-3 and age was analyzed by the GraphPad prism program, and the correlation was presented as Pearson r, correlation coefficient. Statistical power values (1- $\beta$ error) were calculated using the $G^{*}$ Power 3.1 program.

\section{Results}

Effects of oxidation of the coating antibody and blocking agent. Fig. 1 is a schematic diagram of the antibody-lectin sandwich assay for detecting glycosylation of serum CA15-3. The basic purpose of the system is to detect specific reactions between glycosyl groups of CA15-3 and the lectin (red box). 
Table I. Binding properties of lectins used in this study. The binding-specificities of lectins were referred from a previous report (15).

\begin{tabular}{|c|c|c|c|}
\hline Abbreviation & Full name & Specificity & $\begin{array}{l}\text { Type of glycan } \\
\text { linkage }\end{array}$ \\
\hline ConA & Concanavalin A & $\alpha$-linked mannose high mannose type glycans & N-linked \\
\hline SNA & Sambucus nigra lectin & Sialic acid attached to galactose in $\alpha-2,6$ linkage & $\mathrm{N}$ - and $\mathrm{O}$-linked \\
\hline PNA & Peanut agglutinin & $\begin{array}{l}\text { Galactose attached to } N \text {-acetylgalactosamine } \\
\text { in } \beta-1,3 \text { linkage }\end{array}$ & O-linked \\
\hline RCA I & Ricinus communis agglutinin I & $\begin{array}{l}\text { Galactose attached to } N \text {-acetylglucosamine } \\
\text { in } \beta-1,4 \text { linkage }\end{array}$ & N-linked \\
\hline HPA & Helix pomatia agglutinin & $N$-acetylgalactosamine & O-linked \\
\hline UEA-I & Ulex europaeus agglutinin I & $\alpha$-linked fucose & $\mathrm{N}$ - and O-linked \\
\hline AAL & Aleuria aurantia lectin & $\begin{array}{l}\text { Fucose linked }(\alpha-1,6) \text { to } N \text {-acetylglucosamine } \\
\text { or fucose linked }(\alpha-1,3) \text { to } N \text {-acetyllactosamine }\end{array}$ & $\mathrm{N}$ - and $\mathrm{O}$-linked \\
\hline LCA & Lens culinaris agglutinin & $\begin{array}{l}\alpha \text {-linked mannose } \alpha \text {-linked fucose attached to the } \\
N \text {-acetylchitobiose portion markedly enhances } \\
\text { affinity }\end{array}$ & $\mathrm{N}$-linked \\
\hline $\mathrm{AOL}$ & Aspergillus oryzae lectin & $\alpha 1,6$-linked fucose & N-linked \\
\hline WFA & Wisteria floribunda Lectin & $\begin{array}{l}\text { Carbohydrate structures terminating in } \\
N \text {-acetylgalactosamine linked } \alpha \text { or } \beta \text { to } \\
\text { the } 3 \text { or } 6 \text { position of galactose }\end{array}$ & NA \\
\hline MAA & Maackia amurensis lectin II & Sialic acid attached to galactose in $\alpha-2,3$ linkage & $\mathrm{N}$ - and $\mathrm{O}$-linked \\
\hline Jacalin & $\begin{array}{l}\text { Jackfruit (Artocarpus } \\
\text { heterophyllus) }\end{array}$ & $\begin{array}{l}\text { Galactose attached to } N \text {-acetylgalactosamine in } \\
\beta-1,3 \text { linkage/mono- or disialylated form of } \\
O \text {-glycan }\end{array}$ & O-linked \\
\hline
\end{tabular}

NA, not available.

Both the anti-CA15-3 monoclonal antibody used as a coating antibody and the normal FBS used as a blocking agent contain large amounts of glycosyl groups (blue box). Therefore, a reaction between the glycans of the coating antibody, FBS and the lectin (blue box) have to be blocked prior to the reaction between glycans of CA15-3 and the lectin (red box).

Twelve types of lectin were used to investigate the lectin-binding properties of the coating antibody and FBS. The binding-specificities of lectins for the glycan structures are shown in Table I. FBS was found to react strongly with Concanavalin A (ConA), Sambucus nigra lectin (SNA), Ricinus communis agglutinin I (RCA I), Maackia amurensis lectin II (MAA) and jacalin (Fig. 2A) while the immobilized anti-CA15-3 monoclonal antibody was found to react strongly with ConA, SNA, RCA I, Aleuria aurantia lectin (AAL), Lens culinaris agglutinin (LCA), Aspergillus oryzae lectin (AOL) and jacalin (Fig. 2B). Oxidation of the coating antibody and FBS with sodium metaperiodate caused a significant reduction in reactivity with the lectins. However, oxidation did not completely block the reactions of the coating antibody and FBS with SNA, MAA and jacalin (red boxes in Fig. 2). Therefore, these three lectins were omitted from subsequent lectin screens.

Selection of a lectin for detecting glycosylation of CA15-3. Fig. 3A reveals the results of a full series of sandwich assays using 9 different lectins. The signal produced with ConA was considerably higher than those produced with the other lectins. There were no reactions when human serum or ConA reactions were omitted (Fig. 3B), confirming that the reaction was specific for glycosylation of CA15-3. ConA has been revealed to recognize mannosylated $N$-glycans (19). Hence our results indicate that the mannosylated $N$-glycans of CA15-3 are the most effective targets for the antibody-lectin sandwich assay.

Linearity of the antibody-lectin sandwich assay. Three serum samples each were selected from benign, stage I and stage III cancers, respectively, to investigate linearity as a function of serum dilution. These serum samples were chosen to yield low, medium and high values of the sandwich assay, respectively. As shown in Fig. 4A, the $r^{2}$ values of all three groups appeared to be high $\left(r^{2}>0.9\right)$. No signal was found when rabbit or bovine serum was used (Fig. 4B), indicating that the sandwich assay is specific for human CA15-3.

Comparison of the results for the different cancer groups. The $N$-glycan levels of CA15-3 in benign breast disease and breast cancer (cases) were assessed with the anti-CA15-3 antibody-ConA sandwich assay. As shown in Fig. 5A, markedly higher values were found in the breast cancers. There was no difference between the normal and benign group in the mannosylated $\mathrm{N}$-glycan level of CA15-3 (Fig. 5B). The 
A

FBS

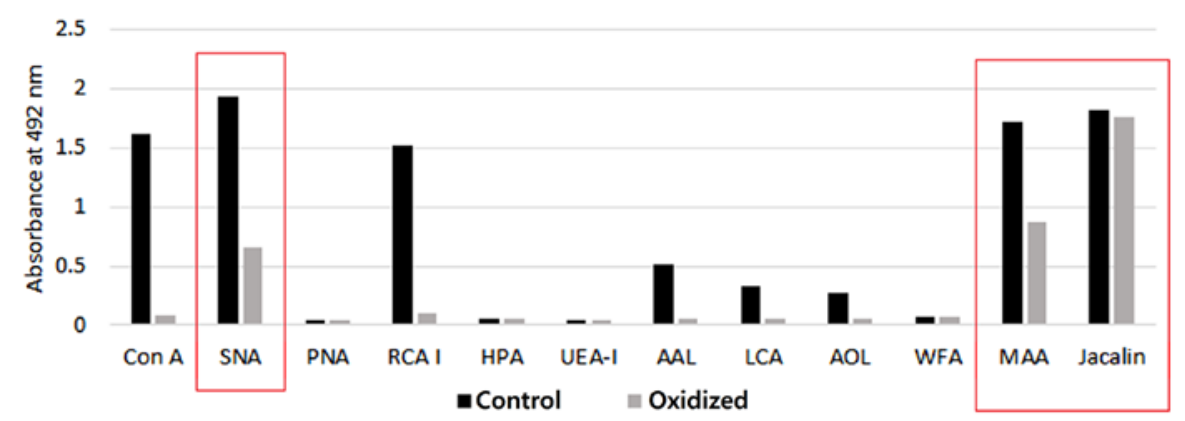

B

Coating Ab

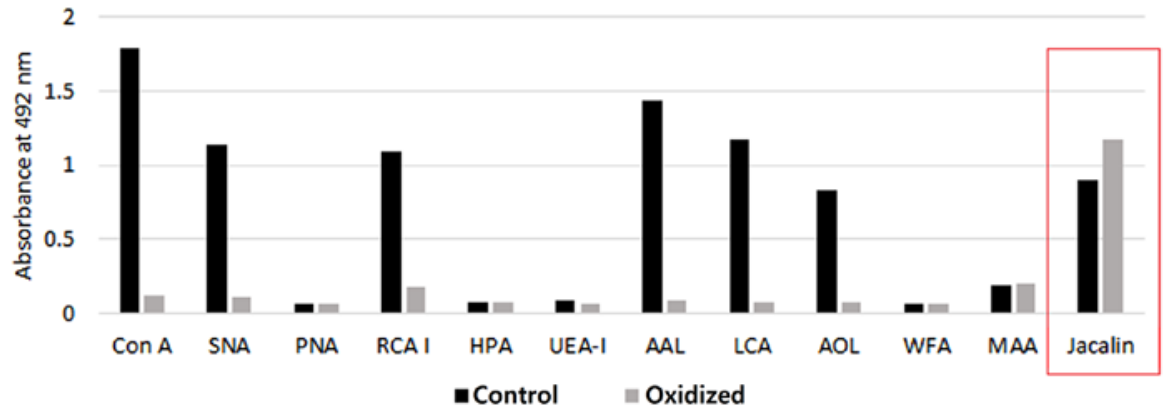

Figure 2. The effect of oxidation on the coating antibody and blocking agent. Immunoplates (96 wells) were coated with (A) FBS or (B) the anti-CA15-3 monoclonal antibody and incubated with oxidation buffer (oxidation) or TBS-T (control). Twelve types of lectins were used. ConA, Concanavalin A; SNA, Sambucus nigra lectin; PNA, Peanut agglutinin; RCA I, Ricinus communis agglutinin I; HPA, Helix pomatia agglutinin; UEA-I, Ulex europaeus agglutinin I; AAL, Aleuria aurantia lectin; LCA, Lens culinaris agglutinin; AOL, Aspergillus oryzae lectin; WFA, Wisteria floribunda lectin; MAA, Maackia amurensis lectin II.

A

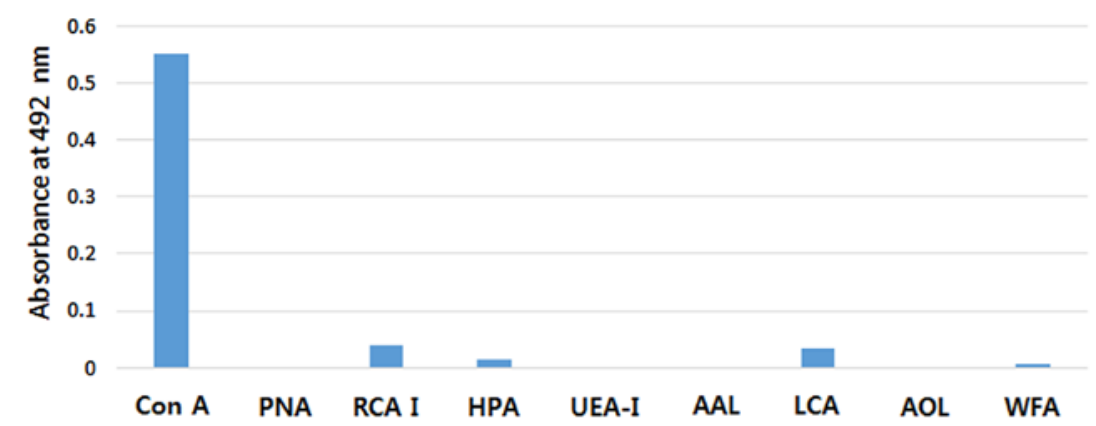

B

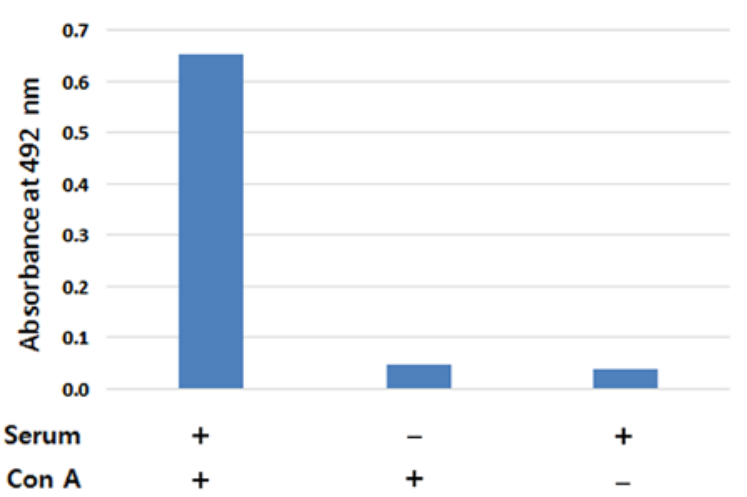

Figure 3. Screening of lectins suitable for detecting serum CA15-3, and the reaction specificity of the antibody-lectin sandwich assay. (A) Antibody-lectin sandwich assays were conducted with the 9 lectins. A mixture of sera of breast cancer stage III $(n=24)$ was used to screen the lectins. (B) Specificity of the anti-CA15-3 antibody-Con A sandwich assay. The value of human serum reaction-omitted condition or Con A reaction-omitted condition was compared with that of a full series reaction. ConA, Concanavalin A; PNA, Peanut agglutinin; RCA I, Ricinus communis agglutinin I; HPA, Helix pomatia agglutinin; UEA-I, Ulex europaeus agglutinin I; AAL, Aleuria aurantia lectin; LCA, Lens culinaris agglutinin; AOL, Aspergillus oryzae lectin; WFA, Wisteria floribunda lectin. 


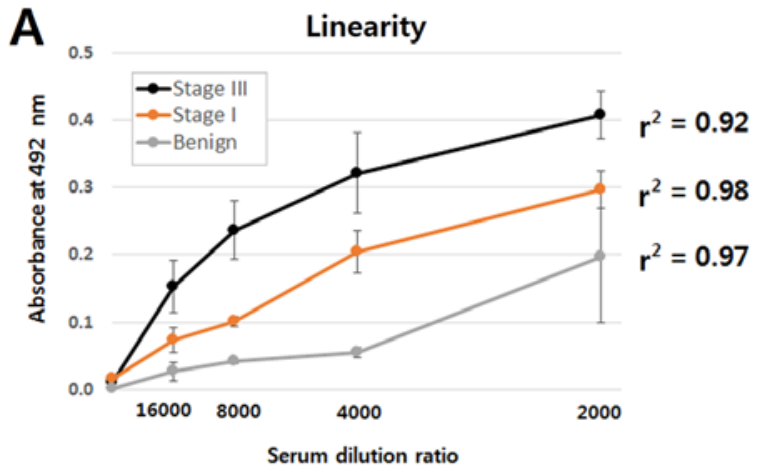

B Specificity

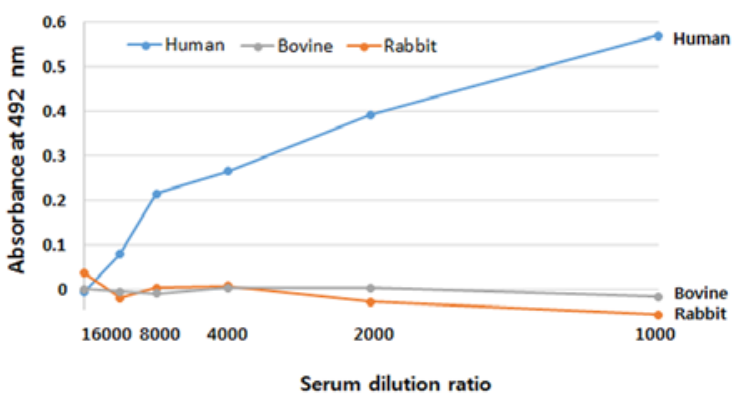

Figure 4. Linearity and specificity of the anti-CA15-3 antibody-ConA sandwich assay. (A and B) The results of the linearity and specificity of the sandwich assay, respectively. (A) Three sera each of benign, stage I and stage III cancers were used to investigate the linearity of the sandwich assay. Serum samples were serially diluted from 1:2,000 to 1:16,000. Data are expressed as the means \pm SEMs. (B) The reactivity of human, rabbit and bovine sera in the sandwich assay were compared. Mixtures of sera of breast cancer stage III $(n=24)$ were used in the human serum reaction. Rabbit anti-human papillomavirus type $16 \mathrm{~L} 1$ serum and FBS were used in the rabbit and bovine serum reactions, respectively. Sera were serially diluted from 1:1,000 to 1:16,000. The sandwich assay was conducted as described in Materials and methods.

A

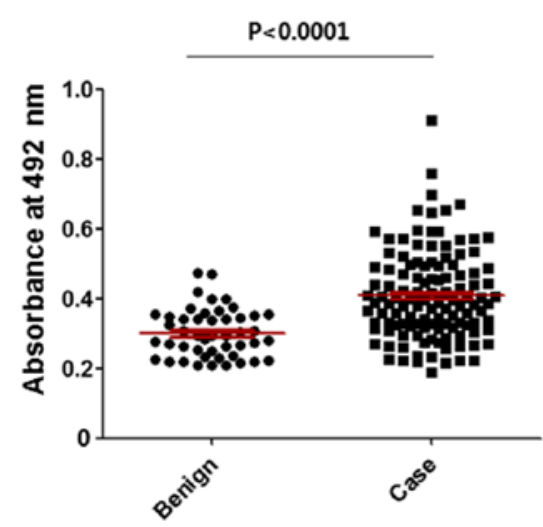

B

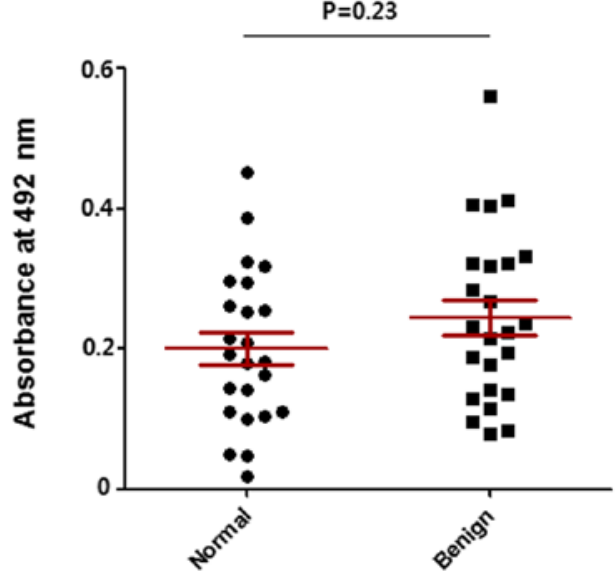

Figure 5. Comparison of the mannosylated $N$-glycan levels between benign and cancer cases. (A) Benign tumors $(\mathrm{n}=47$ ) and all breast cancers including stages 0, I, IIA, IIB and III ( $\mathrm{n}=128)$. (B) Normal $(\mathrm{n}=24)$, benign $(\mathrm{n}=24)$. P-values were calculated using the Mann-Whitney U test. Data are expressed as the means \pm SEMs.

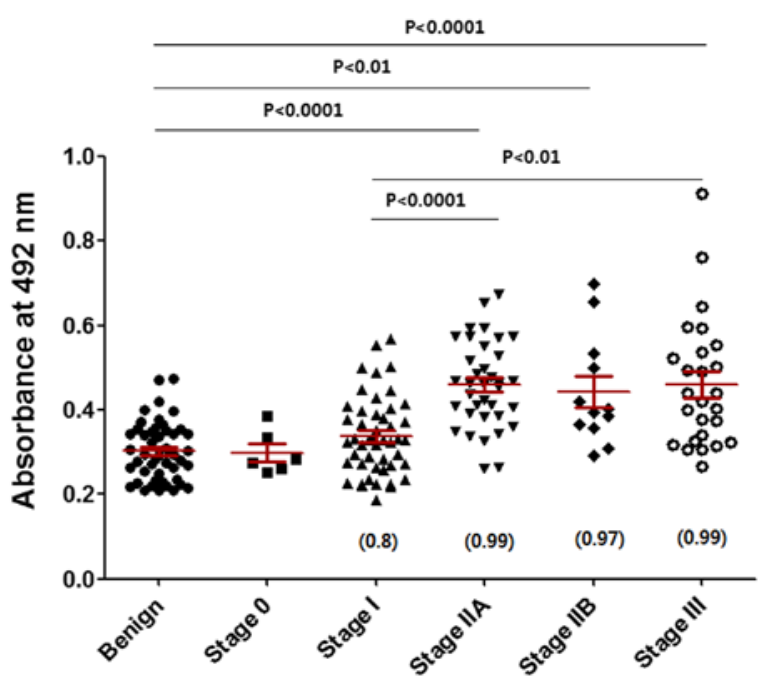

Figure 6. Comparison of the mannosylated $N$-glycan levels between benign tumors and breast cancers of stage 0 , I, IIA, IIB and III. P-values were calculated using the Kruskal-Wallis test. Benign $(n=47)$, stage $0(n=6)$, stage I $(n=48)$, stage IIA $(n=36)$, stage IIB $(n=12)$ and stage III $(n=26)$. Data are expressed as the means \pm SEMs. The numbers in the parentheses are power values (1- $\beta$ error) compared to the benign group. level of CA15-3 appeared to increase with increasing stage of breast cancer (Fig. 6). Mean age of the benign group was 40 , and those of breast cancer stage 0 , I, IIA, IIB and III were $49,51,50,55$ and 54 , respectively. No correlation was found between age and the mannosylated $N$-glycan level of CA15-3 in each group (Table II). Fig. 7 reveals the ROC curves obtained from Fig. 6. Sensitivity, specificity and AUC values in discriminating breast cancer stage I, IIA, IIB or III from benign disease were calculated from three independent assays. The specificity, sensitivity and AUC values were found to be higher when discriminating stage IIA, IIB and III from benign (Table III).

No differences were found between the groups in a sandwich ELISA using two different anti-CA15-3 antibodies rather than the CA15-3 antibody-ConA combination (Fig. 8). The trend in the CA15-3 level between groups in the sandwich ELISA was similar to that reported previously (7). This result indicates that the increased response of the cancer samples in the antibody-lectin sandwich assay is due to increased $\mathrm{N}$-glycosylation of CA15-3 rather than to an increased concentration of CA15-3. This inference was confirmed by a Western 
Table II. Correlation between mannosylated N-glycan levels of CA15-3 and age in each group.

\begin{tabular}{lcccccc}
\hline & Benign $(\mathrm{n}=47)$ & Stage $0(\mathrm{n}=6)$ & Stage I $(\mathrm{n}=48)$ & Stage IIA $(\mathrm{n}=36)$ & Stage IIB $(\mathrm{n}=12)$ & Stage III $(\mathrm{n}=26)$ \\
\hline Range of age & $19-74$ & $25-76$ & $29-78$ & $29-75$ & $33-83$ & $36-78$ \\
Mean age & 40 & 49 & 51 & 50 & 55 & 54 \\
Pearson, $\mathrm{r}$ & -0.09 & -0.38 & -0.12 & 0.22 & 0.02 & -0.23 \\
\hline
\end{tabular}
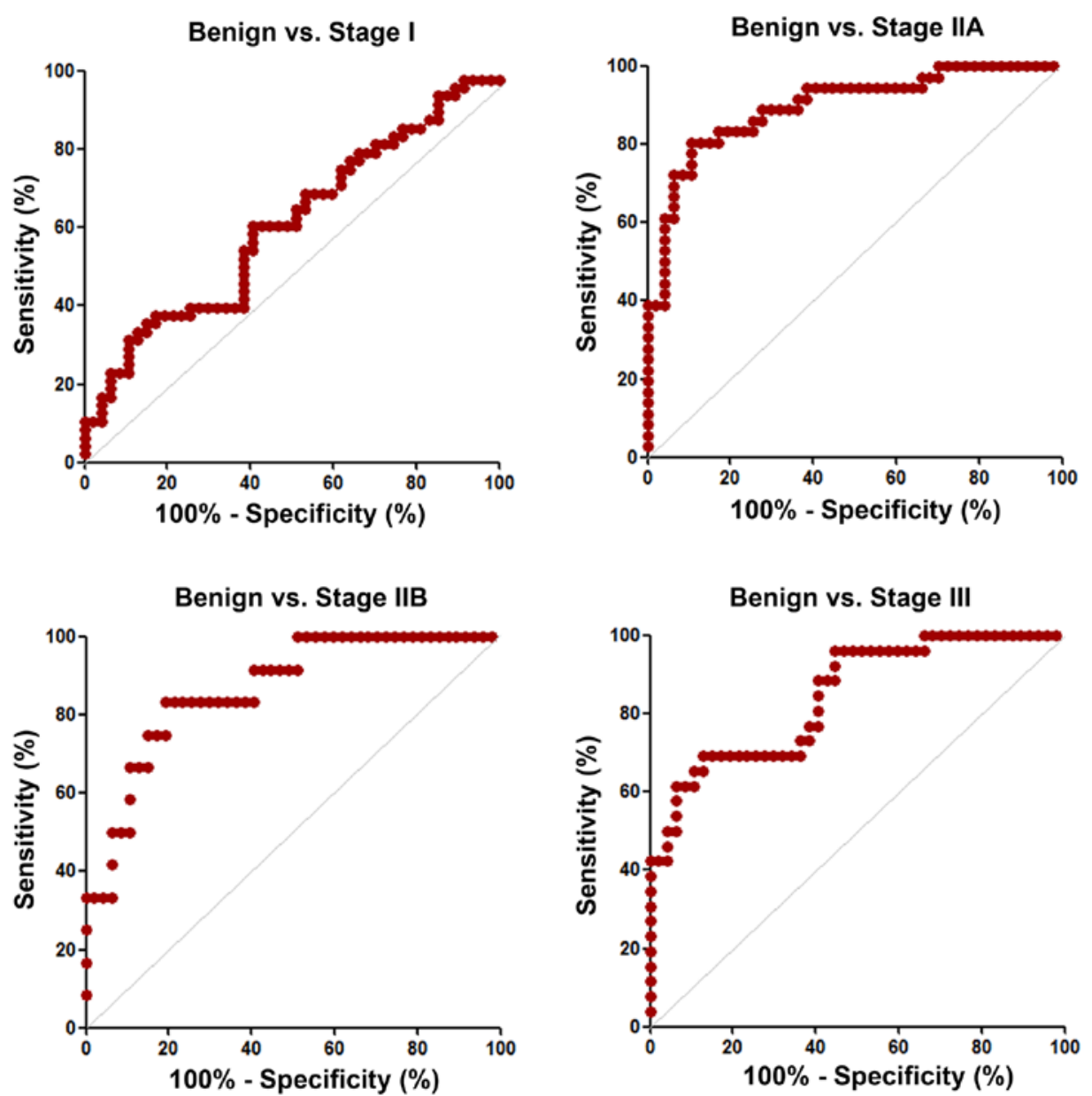

Figure 7. ROC curves for discriminating breast cancer stages I, IIA, IIB and III from benign tumors. The ROC curves were obtained from the data in Fig. 6.

A

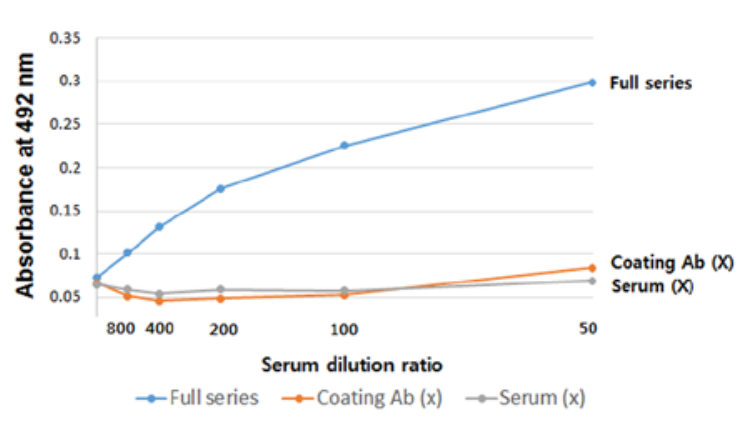

B

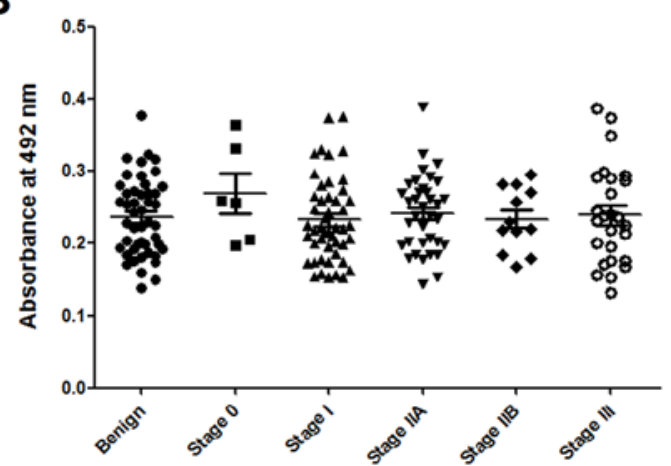

Figure 8. Comparison of the levels of CA15-3 between groups in the sandwich ELISA. Mouse anti-CA15-3 monoclonal antibody (50 ng/well) and rabbit anti-CA15-3 polyclonal antibody (1:3,000 dilution ratio) were used as capture and detection antibodies, respectively. (A) The linear response of the ELISA as a function of serum dilution. The serum used for panel A was a mixture of stage III breast cancers ( $n=24)$. Serum (X) and Coating Ab $(X)$ represent the results obtained when the serum and coating antibody reaction, respectively, were omitted. (B) The values obtained in the sandwich ELISA when a 1:100 serum dilution was used. Data are expressed as the means \pm SEMs. 


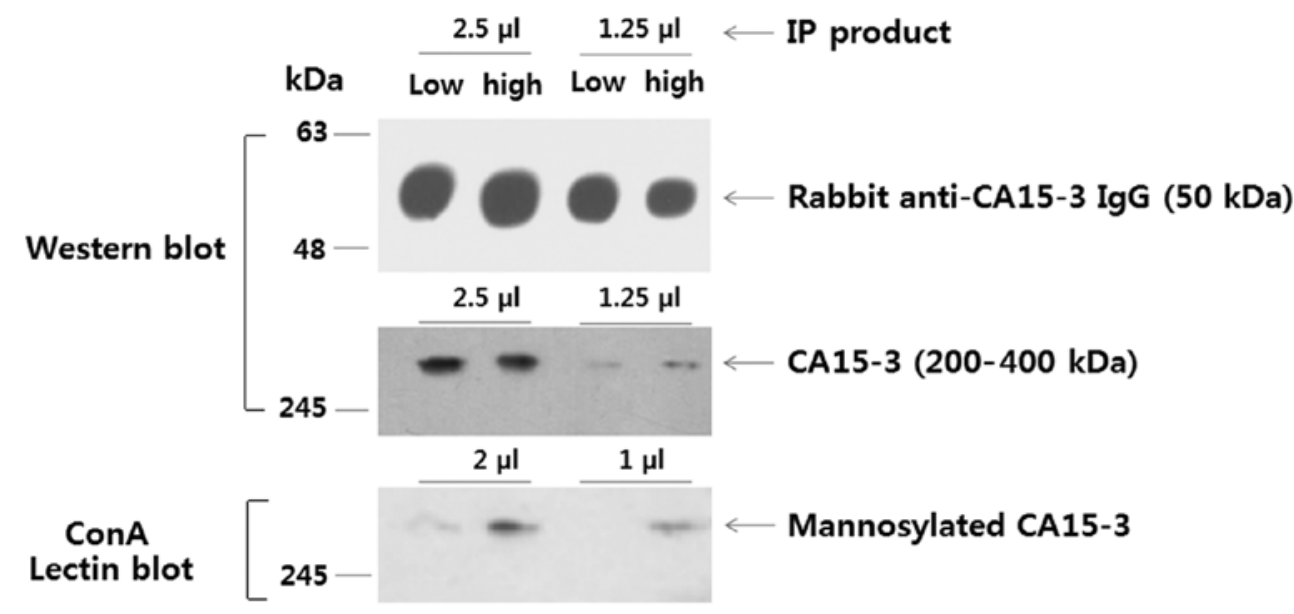

Figure 9. Western blots for the detection of CA15-3, and Con A blots for the detection of mannosylated $N$-glycans on CA15-3. CA15-3 was recovered from low and high serum samples by immunoprecipitation. The low and high samples were the serum mixtures yielding low and high values, respectively, in the sandwich assays of Fig. 6 (benign and breast cancer stage III groups, respectively). The band intensities of the rabbit anti-CA15-3 IgG used for immunoprecipitation (loading control) were checked to ensure that the same amounts of immunoprecipitate were used in each assay. Images for the detection of rabbit anti-CA15-3 IgG, CA15-3 and mannosylated CA15-3 were obtained from separated blots.

Table III. Sensitivity, specificity and AUC values when discriminating stage I, IIA, IIB or III from benign. Three independent assays were carried out and sensitivity, specificity and AUC values were calculated from the assays.

\begin{tabular}{lccc}
\hline & Sensitivity & Specificity & AUC \\
\hline Benign vs. stage I & $63 \pm 30$ & $69 \pm 23$ & $0.64 \pm 0.05$ \\
Benign vs. stage IIA & $77 \pm 4$ & $75 \pm 15$ & $0.78 \pm 0.12$ \\
Benign vs. stage IIB & $69 \pm 17$ & $86 \pm 12$ & $0.79 \pm 0.07$ \\
Benign vs. stage III & $80 \pm 10$ & $65 \pm 20$ & $0.75 \pm 0.08$
\end{tabular}

AUC, area under the curve.

blot detecting CA15-3 and a ConA blot detecting $N$-glycans on CA15-3 (Fig. 9). In summary, our results indicated that the anti-CA15-3 antibody-ConA sandwich assay could be valuable for screening early breast cancers.

\section{Discussion}

CA15-3 (MUC1) is an extensively $O$-glycosylated and moderately $N$-glycosylated protein, and glycosylation is responsible for $50-90 \%$ of its total molecular weight (6). In the present study, an anti-CA15-3 antibody-ConA sandwich assay system was designed to detect the N-glycosylation of serum CA15-3. A total of nine lectins that bind to $O$-glycans or $N$-glycans (20), were tested to investigate their strength binding to captured CA15-3 (Fig. 3A). Unexpectedly, only ConA exhibited strong reactivity towards CA15-3. This implies that the accessibilities of the lectins other than ConA are hindered in some way. Generally, mucin-type glycoproteins and even purified mucin (21) exhibit a tendency to form aggregates or gels (22). Moreover, CA15-3 possesses a self-aggregation domain (23). These properties of mucin-type glycoproteins are likely to mask the glycans and hinder the access of lectins. Lectins have poor access to heavily $O$-glycosylated serum glycoproteins and their access is improved by perchloric acid treatment (24). The conformations and sizes of mucin-type glycoproteins are dependent on factors such as $\mathrm{pH}$ and ionic strength (21). Therefore, further investigation of the conformational changes of CA15-3 as a function of physical conditions may extend the utility of lectins.

The $O$-glycan profiles of CA15-3 from breast milk, urine and breast carcinoma cell lines have been investigated (25-27). The glycan of CA15-3 in culture supernatants and cell lysates of breast carcinoma cell lines was found to have a truncated precursor structure (25-28). On the other hand, little attention has been paid to the $N$-glycan profiles of CA15-3 during carcinogenesis. In the present study, the mannosylated $N$-glycosylation level of CA15-3 increased with increasing stage of breast cancer (Fig. 6). A high mannose type of $N$-glycan is synthesized early in glycan biosynthesis and the glycan is then modified by substitution or addition of other sugars such as sialic acid and galactose (29). Analysis of the $N$-glycan profiles of mouse and human sera using total serum glycoprotein fractions (30) revealed a large number of high mannose-type glycans containing nine mannoses in the glycoproteins from both mice and humans with breast cancer. Therefore, premature termination of the glycosylation pathway is thought to be a critical indicator of the progression of breast cancer. We suggest that alterations of the $N$-glycosylation of CA15-3 would merit examination in future studies of breast carcinogenesis.

The current ELISA system for measuring the serum CA15-3 level (115D8 and DF3 system) allows serum dilutions of 1:50-1:100, whereas our antibody-lectin sandwich assay system detects serum dilutions exceeding 1:2,000 (Fig. 4A). Therefore, the sensitivity of our system is significantly higher than that of the current ELISA. This is because the signal is increased by the abundant glycosylation of CA15-3. This antibody-lectin sandwich assay system appears to have great potential for detecting glycosylation levels of serum glycoproteins in patients with cancers. 
Limited success has been achieved to date with antibody-lectin sandwich systems using clinical samples $(16,17)$. Glycosylation of the coating antibody and blocking agent tend to restrict use of lectins (Fig. 2). Moreover, non-specific reactions of serum proteins are important consideration. Concurrently, FBS or skim milk, which contain large amounts of glycoprotein, are much more effective in blocking the non-specific reactions of human serum protein in immunoassays than BSA (non-glycosylated protein) (31). The choice of blocking agent is therefore not straightforward. In the present study, oxidation of the coating antibody or FBS was not effective in blocking the binding of SNA, MAA and jacalin, which target sialylation (Fig. 2). Changes in sialylation levels have been detected in the sera of cancer patients (32) and deserve further study. In addition, the use of non-glycosylated antibodies and a blocking agent in antibody-lectin sandwich assays could extend the use of lectins. One research group has performed antibody-lectin assays using a Fab fragment of anti-haptoglobin antibody and AAL to detect fucosylated haptoglobin in the sera of patients with colorectal cancer (33). Another research team has suggested that the synthetic blocking agent polymer, polyvinyl alcohol, is effective in lectin-based assays and avoids the background signals caused by glycoprotein-based blocking agents (34).

Limitations of our study are the low number of serum samples examined, as well as the absence of data on metastatic breast cancer and changes in glycosylation levels after surgery. We anticipate that studies with a larger number of samples will extend the utility of the sandwich assay for measuring the glycosylation level of CA15-3 in breast cancers and the use of this assay as a prognostic marker.

Aberrant glycosylation during cancer progression has been found in several types of serum glycoproteins such as prostate specific antigen, carcinoma antigen 125, carcinoembryonic antigen and human chorionic gonadotropin $\beta$ subunit (14). Since almost $60 \%$ of serum proteins are glycosylated and more than 100 types of lectin are commercially available (35), the antibody-lectin sandwich assay platform has great potential for the early detection of cancers. We believe that the present study provides a foretaste of a novel and promising type of a high-throughput cancer screening system.

\section{Acknowledgements}

We thank Mei Ling Xu for preparing the samples used in this study and Julian D. Gross (Oxford University, UK) for English editing.

\section{Funding}

The present study was supported by a grant of the Korean Health Technology R\&D Project, Ministry of Health and Welfare, Republic of Korea (HI12C0050). URL: https://www. htdream.kr/.

\section{Availability of data and materials}

The datasets used during the present study are available from the corresponding author upon reasonable request.

\section{Authors' contributions}

JWC, HJK, BIM, JWL and HJK conceived and designed the study. JWC and HJK wrote the paper. BIM and JWL collected specimens. JWC, HJK and YIJ carried out experiments. HJK is responsible for the integrity of the work as a whole.

\section{Ethics approval and consent to participate}

The present study was carried out with the approval of the Ewha Womans University Mokdong Hospital Institutional Review Board (Seoul, Republic of Korea) and was conducted in accordance with the Declaration of Helsinki. Serum samples of patients were collected after obtaining written informed consents.

\section{Consent for publication}

Not applicable.

\section{Competing interests}

The authors declare that they have no competing interests.

\section{References}

1. InternationalWCR: Breastcancerstatistics.accessed on 16January 2015 http://www.wcrf.org/int/cancer-facts-figures/data-specificcancers/breast-cancer-statistics.2015.

2. Torre LA, Bray F, Siegel RL, Ferlay J, Lortet-Tieulent J and Jemal A: Global Cancer Statistics, 2012. Ca Cancer J Clin 65: 87-108, 2015.

3. Dumalaon-Canaria JA, Hutchinson AD, Prichard I and Wilson C: What causes breast cancer? A systematic review of causal attributions among breast cancer survivors and how these compare to expert-endorsed risk factors. Cancer Causes Control 25: 771-785, 2014.

4. Misek DE and Kim EH: Protein biomarkers for the early detection of breast cancer. Int J Proteomics 2011: 343582, 2011.

5. Chou CP, Peng NJ, Chang TH, Yang TL, Hu C, Lin HS, Huang JS and Pan HB: Clinical roles of breast 3T MRI, FDG PET/CT, and breast ultrasound for asymptomatic women with an abnormal screening mammogram. J Chin Med Assoc 78: 719-725, 2015.

6. Nath S and Mukherjee P: MUC1: A multifaceted oncoprotein with a key role in cancer progression. Trends Mol Med 20: 332-342, 2014.

7. Duffy MJ, Evoy D and McDermott EW: CA 15-3: Uses and limitation as a biomarker for breast cancer. Clin Chim Acta 411: 1869-1874, 2010.

8. Kufe D, Inghirami G, Abe M, Hayes D, Justi-Wheeler H and Schlom J: Differential reactivity of a novel monoclonal antibody (DF3) with human malignant versus benign breast tumors Hybridoma 3: 223-232, 1984.

9. Duffy MJ, Shering S, Sherry F, McDermott E and O'Higgins N: CA 15-3: A prognostic marker in breast cancer. Int $\mathbf{J}$ Biol Markers 15: 330-333, 2000.

10. Harris L, Fritsche H, Mennel R, Norton L, Ravdin P, Taube S, Somerfield MR, Hayes DF and Bast RC Jr; American Society of Clinical Oncology: American society of clinical oncology 2007 update of recommendations for the use of tumor markers in breast cancer. J Clin Oncol 25: 5287-5312, 2007.

11. Munkley J and Elliott DJ: Hallmarks of glycosylation in cancer. Oncotarget 7: 35478-35489, 2016.

12. Storr SJ, Royle L, Chapman CJ, Hamid UMA, Robertson JF, Murray A, Dwek RA and Rudd PM: The O-linked glycosylation of secretory/shed MUC1 from an advanced breast cancer patient's serum. Glycobiology 18: 456-462, 2008.

13. Lavrsen K, Madsen CB, Rasch MG, Woetmann A, Odum N, Mandel U, Clausen H, Pedersen AE and Wandall HH: Aberrantly glycosylated MUC1 is expressed on the surface of breast cancer cells and a target for antibody-dependent cell-mediated cytotoxicity. Glycoconj J 30: 227-236, 2013. 
14. Kuzmanov U, Kosanam H and Diamandis EP: The sweet and sour of serological glycoprotein tumor biomarker quantification. BMC Med 11: 31, 2013.

15. Hashim OH, Jayapalan JJ and Lee CS: Lectins: An effective tool for screening of potential cancer biomarkers. Peer J 5: e3784, 2017.

16. Madiyalakan R, Kuzma M, Noujaim AA and Suresh MR: An antibody-lectin sandwich assay for the determination of CA125 antigen in ovarian cancer patients. Glycoconj J 13: 513-517, 1996.

17. Bamrungphon W, Prempracha N, Bunchu N, Rangdaeng S, Sandhu T, Srisukho S, Boonla C and Wongkham S: A new mucin antibody/enzyme-linked lectin-sandwich assay of serum MUC5AC mucin for the diagnosis of cholangiocarcinoma. Cancer Lett 247: 301-308, 2007.

18. Kim HJ and Lee SJ: Antibody-based enzyme-linked lectin assay (ABELLA) for the sialylated recombinant human erythropoietin present in culture supernatant. J Pharm Biomed Anal 48: 716-721, 2008.

19. Cummings RD and Etzler ME: Antibodies and lectins in glycan analysis, 2009.

20. Zhang L, Luo S and Zhang BL: The use of lectin microarray for assessing glycosylation of therapeutic proteins. MAbs 8 : 524-535, 2016.

21. Bansil R and Turner BS: Mucin structure, aggregation, physiological functions and biomedical applications. Curr Opin Colloid In 11: 164-170, 2006.

22. Taylor C, Allen A, Dettmar PW and Pearson JP: The gel matrix of gastric mucus is maintained by a complex interplay of transient and nontransient associations. Biomacromolecules 4: 922-927, 2003.

23. Mahanta S, Fessler SP, Park J and Bamdad C: A minimal fragment of MUC1 mediates growth of cancer cells. PLoS One 3 : e2054, 2008.

24. Lee CS, Taib NAM, Ashrafzadeh A, Fadzli F, Harun F, Rahmat K, Hoong SM, Abdul-Rahman PS and Hashim OH: Unmasking heavily o-glycosylated serum proteins using perchloric acid: Identification of serum proteoglycan 4 and protease $\mathrm{c} 1$ inhibitor as molecular indicators for screening of breast cancer. PLoS One 11: e149551, 2016.

25. Bhavanandan VP, Zhu Q, Yamakami K, Dilulio NA, Nair S, Capon $\mathrm{C}$, Lemoine $\mathrm{J}$ and Fournet B: Purification and characterization of the MUC1 mucin-type glycoprotein, epitectin, from human urine: Structures of the major oligosaccharide alditols. Glycoconj J 15: 37-49, 1998.
26. Hanisch FG, Stadie TRE, Deutzmann F and PeterKatalinic J: MUC1 glycoforms in breast cancer - Cell line T47D as a model for carcinoma-associated alterations of O-glycosylation. Eur J Biochem 236: 318-327, 1996.

27. Brockhausen I, Yang JM, Burchell J, Whitehouse C and Taylor-papadimitriou J: Mechanisms underlying aberrant glycosylation of muc1 mucin in breast-cancer cells. Eur J Biochem 233: 607-617, 1995.

28. Hanson RL and Hollingsworth MA: Functional consequences of differential o-glycosylation of MUC1, MUC4, and MUC16 (downstream effects on signaling). Biomolecules 6: E34, 2016.

29. Stanley P: Golgi glycosylation. Cold Spring Harb Perspect Biol 3: a005199, 2011.

30. de Leoz ML, Young LJ, An HJ, Kronewitter SR, Kim J, Miyamoto S, Borowsky AD, Chew HK and Lebrilla CB: High-mannose glycans are elevated during breast cancer progression. Mol Cell Proteomics 10: M110, 2011.

31. Biocompare:Bench tips:tips forreducingelisa background.http:// www.biocompare.com/Bench-Tips/122704-Tips-for-ReducingELISA-Background/2012.

32. Kirwan A, Utratna M, O'Dwyer ME, Joshi L and Kilcoyne M: Glycosylation-based serum biomarkers for cancer diagnostics and prognostics. Biomed Res Int 2015: 490531, 2015.

33. Takeda Y, Shinzaki S, Okudo K, Moriwaki K, Murata K and Miyoshi E: Fucosylated haptoglobin is a novel type of cancer biomarker linked to the prognosis after an operation in colorectal cancer. Cancer 118: 3036-3043, 2012.

34. Thompson R, Creavin A, O'Connell M, O'Connor B and Clarke P: Optimization of the enzyme-linked lectin assay for enhanced glycoprotein and glycoconjugate analysis. Anal Biochem 413: $114-122,2011$.

35. Sharon $\mathrm{N}$ and Lis H: History of lectins: From hemagglutinins to biological recognition molecules. Glycobiology 14: 53R-62R, 2004.

This work is licensed under a Creative Commons Attribution-NonCommercial-NoDerivatives 4.0 International (CC BY-NC-ND 4.0) License. 